Available online @ https://jiem.jnnce.ac.in https:www.doi.org/10.37314/JJEM.2020.040207 Indexed in International Scientific Indiexing (ISI) Impact factor: 1.025 for 2018-19 Published on: 30 March 2020

\title{
An Efficient Hybrid Multicast Protocol for Manet
}

\author{
Kantharaj S P1*, Samarth $\mathbf{K}^{2}$ \\ ${ }^{1 *}$ Department of Electronics and Communication Engineering, JIT, Davangere, India \\ ${ }^{2}$ Department of Electronics and Communication Engineering, STJIT, Ranebennur, India \\ kantharajsp@gmail.com
}

\begin{abstract}
Routing protocols for MANET have to face the challenge of frequently changing topologies. Conventional proactive and reactive type routing protocols are less efficient under these circumstances. Hybrid protocol named Zone Routing Protocol clubs the advantages of the former protocols by maintaining an up to date topological map. Several virtual architectures are being used for better robust and scalable membership management without maintenance of state information. A Robust and Scalable Geographic Multicast protocol is proposed in this paper forwards the control messages and data packets without explicitly creating and maintain a tree structure. A predefined and properly organized source tracking mechanism is designed to exclude flooding of the MANET source information. Virtual networks are deployed and analyzed for different number of nodes. Our simulation results and comparison shows that proposed protocol offers higher, throughput, lesser drop and lower control over head with even with varying network sizes, moving speeds and node densities.
\end{abstract}

Keywords: Manet, Multicast Protocol, Hybrid Routing, Zone structure

\section{Introduction}

Ad hoc network is a multi-hop wireless network and a mobile ad-hoc network is a self- configuring network of mobile routers connected by wireless links. In MANET, wireless devices could self-configure in the form of an arbitrary topology. In MANET, the network topology changes frequently as the nodes are being connected and disconnected rapidly and unpredictably. Because of this dynamic characteristic MANETs, conventional routing protocols are not viable. Due to mobility of nodes, the wireless links in the network are much error prone and may fail down [1] [3]. Multicast is a way in which packets are delivered from source to many nodes. For, MANET it is quite challenge to support the reliable and scalable multicasting with the changing topologies [1] [5].

\section{Related Work}

Multicast Ad-hoc On-demand Distance Vector (MAODV) protocol is based on reactive tree routing was used and found inefficient. In larger MANETs, it is difficult to create and maintain tree structure, transmission may not be reliable if the tree connection were break. Another mesh based protocol, On Demand Multicast Routing (ODMRP) applies reactive protocol procedures to configure routes. Here, a hybrid Robust and Scalable Geographic Multicast (RSGM) protocol is discussed. RSGM is a zone based routing with geographical squares are considered as zones. Proposed protocol is scalable to group and network size [2] [5] [6]. 


\section{Robust and Scalable Geographic Multicast Protocol (RSGM)}

Hybrid protocols forwards data by finding the route in layered and/or in hierarchical passion. Proposed protocol incorporates a two-tier node membership management and data packets forwarding structure at the lower tier. A virtual zone structure is built based on position information. Zone leader is elected on demand basis. Zone leader manages the group membership and collects the positions of the member nodes in its zone.

At the upper tier, through a virtual reversetree-based structure, zone leaders directly report the zone membership to sources. As a special case, if this leader is unaware of the position or addresses of the source node then the leader can obtain the position information from the source home.

As this two tier procedure, source node multicasts data packets to the zones and packets reach member zone. Now, zone leader forwards the packets through the virtual reverse-tree structure to the nodes of member zone. Zone structure is shown in figure 1.

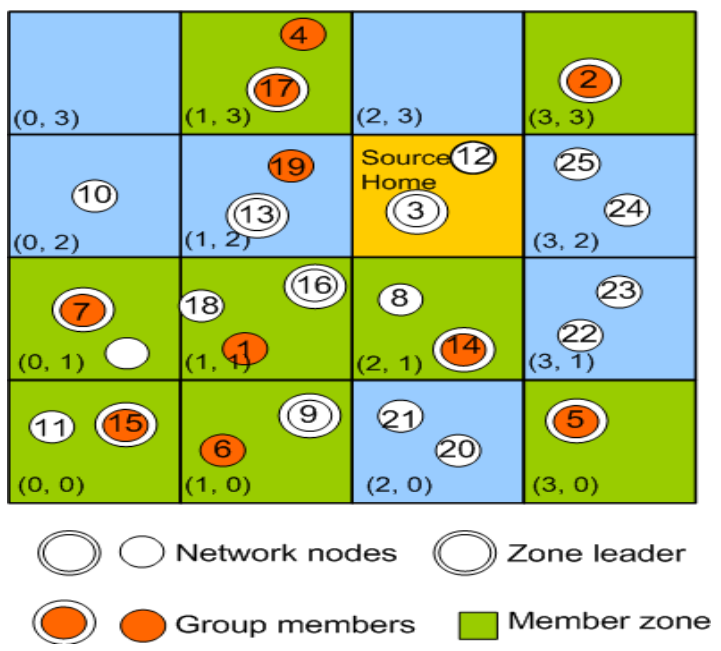

Figure 1: Zone Structures
Notations and references to be used for zone routing are:

pos: mobile node's position - indicated by pos $(\mathrm{x}, \mathrm{y})$. Zone: Geographical network terrain is divided into squares.

sHome: an identified zone in the network which is elected as Source Home (sHome).

mZone: zone with group members is treated as Member zone.

non_mZone: a zone without intended nodes

zID: The address of a multicast group.

zldr: Ellected Zone leader.

\section{A. Virtual Zone Construction:}

The concept of zone is calculated based on a reference point and is virtual. Construction of zone structure independent of shape of the network region with simplicity in locating and maintain zone. Virtual zones are the references for the member nodes. At network initialization stage, zone is set to origin located at (Xo, Yo). Zone size is defined as length of a side of the zone. Every zone is identified by its own ID (zID) and every node calculates positional value $(a, b)$ from its pos from below equations.

$$
a=\frac{x-x_{0}}{\text { zonesize }} \quad \text { and } b=\frac{y-y_{0}}{\text { zonesize }}
$$

Assumed positive IDs will help to locate a zone. A packet destined to a zone will be first forwarded towards its centre. The centre position $(\mathrm{Xc}, \mathrm{Yc})$ is found as:

$$
\begin{aligned}
& \text { Xcenter }=\mathrm{Xo}+(\mathrm{a}+0.5) \times \text { zonesize } \\
& \text { Ycenter }=\mathrm{Yo}+(\mathrm{b}+0.5) \times \text { zonesize }
\end{aligned}
$$




\section{B. Election of Member Zone Leader}

Zone leaders are elected only when the zone has group member and zIDs controls management overhead. If a group member $M$ moves to new zone and zone leader (zLdr) is unknown, $M$ queries a neighbour node for zLdr. During the failure of getting zLdr information, $M$ will flood a LEADER message into the zone and announce itself as zLdr.

In the case of existence of two leaders in single zone then the leader with larger ID will referred as zLdr. In every time interval refresh (Intval) zLdr floods a LEADER to announce its leadership. If no LEADER message is received longer than 2 times of Intval refresh, random member node waits a random period and then makes self-announce as zLdr.

There are two issues which need to be addressed to enhance the protocol functionality.

1. Zone management: This issue accounts for the strategy to elect zone leader with mobile nodes and zone leader maintenance.

2. Empty-zone problem: This issue accounts for Source-Home consideration and maintenance of Nonmember zone.

\section{A. Multicast Packet Delivery}

The flow for the data packets delivery is in conjunction with the following steps:

$>$ Multicast session initiation and termination

$>$ Multicast group joining \& leaving

$>$ Multicast packet transmission

From source, data packets are distributed through virtual tree to the member zones. If the immediate nodes are Intermediate nodes then, the zones except destination zone uses inter-zone routing table. Once the data packets reach the zone leader, then they are delivered to the destination nodes of mZone using an intra-zone routing table.

\section{Performance Evaluation}

The position information is used in RSGM. This results in reduced in maintenance overhead and leads to more robust multicast forwarding even in the case of changing topology. Performance of RSGM is analysed in terms of throughput (figure 2), packet delivery ratio (figure 3) and control overhead (figure 4). RSGM achieves more delivery ratio for different range of group sizes when compared to MAODV and ODMRP.

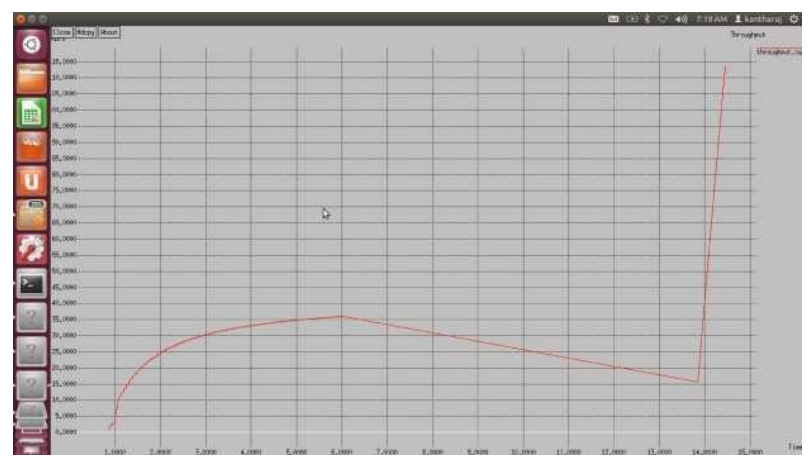

Figure 2: Throughput of RSGM

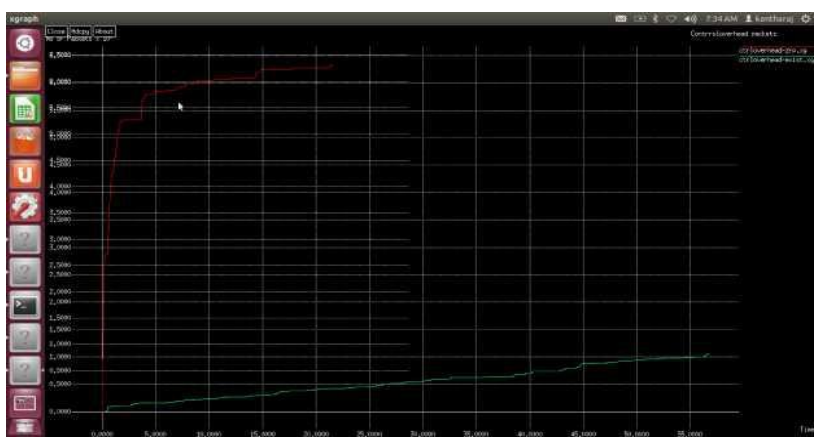

Figure 3: Comparison of Packet Delivery Ratio

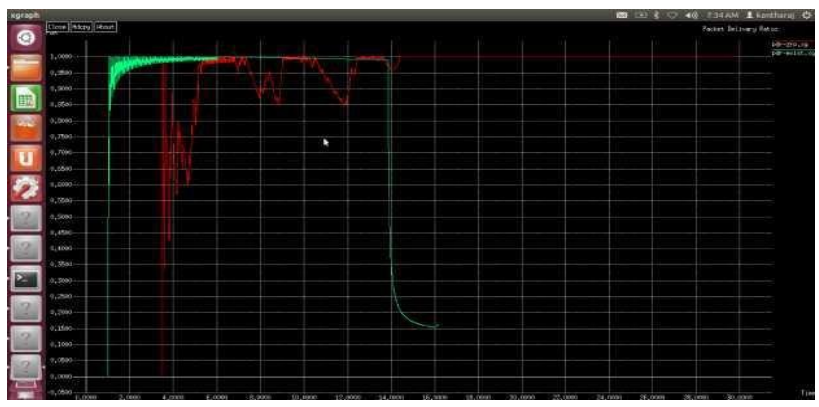

Figure 4. Comparison of Control Overhead 


\section{Conclusion}

A hybrid based Robust and Scalable Geographic Multicast protocol for MANETs is proposed and performance metrics are analysed using NS2. Two-tier infrastructure and virtual zone based forwarding of packets achieves Scalable membership of nodes. By means of simulation results it is obvious that the RSGM protocol has higher packet delivery ratio and lower control over head with better throughput compared with proactive and reactive protocols. The simulation results are also true for the MANETs under the consideration of varying moving speeds, node densities, group sizes and network range.

\section{References}

1. K.Kavitha, and Dr. S. Nagarajan, Efficient Multicasting In MANET Using RSGM Technique, International Journal of Power Control Signal and Computation, Vol. 3. No.1, Jan-Mar 2012, ISSN: 0976-268X 30-35.

2. Shapour joudi Begdillo and Hekmat Mohamamd zadeh, Stable Route Selection in ODMRP with Energy Based Strategy, 2010. Proceedings of the IEEE 21st International Symposium on Personal, Indoor and Mobile Radio Communications, PIMRC 2010, Istanbul, Turkey, 26-29 September 2010,
[3] X. Xiang, Z. Zhou, and X. Wang. Robust and Scalable Geographic Multicast Protocol for Mobile Ad Hoc Networks, Proceedings of the $26^{\text {th }}$ International Conference on Computer Communications IEEE, Anchorage, AK, USA (327) 2007.

[4] Haas, Zygmunt J., Pearlman, Marc R, The Performance of Query Control Schemes for the Zone Routing Protocol, IEEE/ACM Transactions on Networking, Vol. 9, Issue. 4, Aug 2001, 427-438, doi: 10.1109/90.944341.

[5] Safaa Laqtib, Khalid El Yassini and Moulay Lahcen Hasnaoui, Performance Evaluation of Multicast Routing Protocols in MANET, ICAISSD, 2019, 847-856.

[6] Bander H, Qarni, Ahmad S. And Mogren, Reliable and Energy Efficient Protocol for MANET Multicasting, Journal of Computer Networks and Communications, Vol.2016. https://doi.org/10.1155/2016/9146168

[7] Murthy and Garcia-Luna-Aceves, An Efficient Routing Protocol for Wireless Networks, Mobile Networks and Applications, volume 1, 1996, 183-197. 